Article

\title{
Tris-(Nitrilotriacetic Acid)-Decorated Polymer Conjugates as Tools for Immobilization and Visualization of His-Tagged Proteins
}

\author{
Jana Beranová ${ }^{1,2}$, Tomáš Knedlík ${ }^{1,3}$, Adéla Šimková ${ }^{1,4}$, Vladimír Šubr ${ }^{5}$, Libor Kostka ${ }^{5}$, \\ Tomáš Etrych ${ }^{5}$, Pavel Šácha ${ }^{1, *(D)}$ and Jan Konvalinka 1,3,* \\ 1 Institute of Organic Chemistry and Biochemistry, The Czech Academy of Sciences, Flemingovo n. 2, \\ 16610 Prague 6, Czech Republic; jana.beranova@uochb.cas.cz (J.B.); tomas.knedlik@uochb.cas.cz (T.K.); \\ adela.simkova@uochb.cas.cz (A.Š.) \\ 2 First Faculty of Medicine, Charles University, Katerinska 32, 12108 Prague 2, Czech Republic \\ 3 Department of Biochemistry, Faculty of Science, Charles University, Hlavova 8, \\ 12843 Prague 2, Czech Republic \\ 4 Department of Organic Chemistry, Faculty of Science, Charles University, Hlavova 8, \\ 12843 Prague 2, Czech Republic \\ 5 Institute of Macromolecular Chemistry, The Czech Academy of Sciences, Heyrovského n. 2, \\ 16206 Prague 6, Czech Republic; subr@imc.cas.cz (V.Š.); kostka@imc.cas.cz (L.K.); etrych@imc.cas.cz (T.E.) \\ * Correspondence: pavelsacha@gmail.com (P.Š.); jan.konvalinka@uochb.cas.cz (J.K.); \\ Tel.: +420-220-183-452 (P.Š.); +420-220-183-218 (J.K.)
}

Received: 17 October 2019; Accepted: 28 November 2019; Published: 1 December 2019

\begin{abstract}
Recombinant proteins are commonly expressed with artificial affinity tags for purification, immobilization and characterization. The most frequently used tag, His-tag, is a sequence of consecutive histidine residues fused to the protein of interest. Specialized small molecules that bind His-tag are primarily used for purification, while antibodies are used for protein analysis. However, various issues may be encountered with the use of antibodies. Low inherent stability, the difficulty of introducing chemical modifications, and often-unreliable batch-to-batch consistency are among the limiting factors that call for better alternatives. Recently described polymer conjugates of $\mathrm{N}$-(2-hydroxypropyl) methacrylamide and low-molecular-weight functional ligands, so-called iBodies, are antibody mimetics capable of replacing antibodies in biochemical applications. We tailored this system for methods utilizing His-tag by accessorizing the polymer carrier with tris-nitrilotriacetic acid targeting ligands. These anti-polyHis iBodies are additionally accessorized with fluorophores, enabling detection, and biotin ligands, enabling immobilization. Here, we characterized anti-polyHis iBodies and explored their use as antibody mimetics. We tested their stability, as well as the influence of different metal mediators and His-tag lengths on binding. With high affinity and stability, iBodies represent a new alternative for immobilization and visualization of His-tagged proteins.
\end{abstract}

Keywords: antibody mimetics; protein immobilization; His-tag; tris-nitrilotriacetic acid (tris-NTA); iBodies; biocompatible polymers; $N$-(2-hydroxypropyl) methacrylamide (HPMA)

\section{Introduction}

Artificial fusion tags introduced to proteins by recombinant DNA technologies have greatly facilitated methods for selective protein purification, characterization and immobilization.

A wide variety of affinity tags are available, among which the so-called His-tag is the most commonly used. This sequence of six to ten consecutive histidine residues (rarely occurring in nature) fused to the N- or C-terminus of recombinant proteins can be used to specifically target the protein of 
interest. Depending on the application, His-tag can be recognized by either matrices functionalized with small molecule binders or specific monoclonal antibodies (mAbs) [1].

His-tag forms a highly selective, yet reversible, complex with small molecule ligands charged with metal cation mediators, which are used to functionalize solid supports. Such interaction provides an efficient means of isolation of proteins from the host system and purification from the complex cellular matrix. The affinity of the His-tag proteins for functionalized surface matrices can be affected by numerous factors. The choice of the chelating agent and metal intermediate involved in coordination play the most significant roles. Various chelating agents are available, but the two compounds used predominantly are nitrilotriacetic acid (NTA) and iminodiacetic acid (IDA). The most commonly used mediator ions include $\mathrm{Co}^{2+}, \mathrm{Ni}^{2+}, \mathrm{Cu}^{2+}$ and $\mathrm{Zn}^{2+}$, which show a preference for nitrogen-rich residues and primarily target the polyhistidine sequence under optimized conditions. The choice of the affinity-mediator agents depends on the purposes of the application. For example, in the case of protein purification, formation of more labile complexes is preferred, whereas in applications such as bio-conjugation or labeling, higher affinities are desirable [2-4].

Although originally (and still primarily) used for protein purification, His-tag also serves as a tool in many other applications, including molecule immobilization and visualization. These biochemical methods make use of monoclonal antibodies (mAbs) that are able to recognize and specifically bind the fusion tag via protein-protein interactions. Such antibodies play crucial roles in immunochemical methods commonly used for protein characterization (ELISA, Western blotting, flow cytometry, immunoprecipitation, confocal fluorescent microscopy, etc.) [5].

However, various issues are encountered with the use of antibodies. The low stability inherent to proteins, difficulty of introducing chemical modifications, and often-unreliable batch-to-batch consistency and target specificity are among the major limitations that can adversely affect the quality of research [6,7]. The fairly young field of antibody mimetics aims to develop alternative, more reliable strategies.

Recently, we described antibody mimetics based on synthetic polymer conjugates, so-called iBodies [8-10]. In the present work, we tailored this polymer-based flexible system to provide a substitute for anti-His antibodies in applications involving His-tag. Inspired by the principles of specific immobilization via small molecules, water-soluble N-(2-hydroxypropyl) methacrylamide (HPMA) copolymer carrier was decorated with tris-nitrilotriacetic acid (tris-NTA) ligands (increasing the affinity for His-tag through NTA multivalency) [11], providing a means of targeting. Furthermore, these anti-polyHis iBodies were accessorized with biotin, which can be used as an affinity anchor to immobilize the conjugates, and fluorophore molecules, which allow facile detection and visualization of the target proteins.

We explored the efficiency of anti-polyHis iBodies as antibody mimetics in various biochemical applications including Western blotting, ELISA and immobilization experiments, and describe their binding behavior and stability. Different modes of detection are presented to highlight the flexibility of the iBody platform. Furthermore, we discuss differences in binding affinities of iBodies charged with two different metal cations $\left(\mathrm{Co}^{2+} \mathrm{vs} . \mathrm{Ni}^{2+}\right)$ and their interactions with two polyhistidine sequences of different lengths.

\section{Results and Discussion}

\subsection{Preparation and Characterization of Anti-polyHis iBody Conjugates}

To obtain well-defined antibody mimetics, controlled reversible addition-fragmentation chain-transfer polymerization technique was used for synthesis of iBodies. This technique allows precise control of molecular weight, keeping the dispersity of the polymer precursor at around 1.2. Molecular weight of the polymer precursors was set to approximately $70 \mathrm{~kg} / \mathrm{mol}$ after optimization in previous studies. Such length of polymer allows sufficient incorporation of comonomers with reactive groups, which corresponds to 50 reactive groups per polymer chain on average. We prepared two 
versions of anti-polyHis iBodies (Figure 1) differing in the conjugated fluorescent probe to provide distinct means of detection. iBody 1 was designed with the fluorophore Cyanine 7.5 (excitation maximum $=788 \mathrm{~nm}$, emission maximum $=808 \mathrm{~nm}$ ) for detection within the near infrared (NIR) region of the light spectrum. For iBody 2, ATTO488 (excitation maximum $=502 \mathrm{~nm}$, emission maximum $=523$ $\mathrm{nm}$ ) was chosen as a more conventional means of detection. As a negative control, we prepared a polymer conjugate accessorized with ATTO488 and biotin ligands but lacking the targeting tris-NTA molecules (iBody 3 ). For conjugation of functional ligands $74 \%$ or $48 \%$, respectively, of reactive groups were used in the case of iBody 1 or iBody 2 , respectively. Remaining reactive groups were depleted with 1-aminopropan-2-ol.

We characterized the iBodies by determining weight-average molecular weights $\left(M_{\mathrm{W}}\right)$, number-average molecular weights $\left(M_{\mathrm{n}}\right)$, and dispersity $(\nexists)$ of the polymer precursors and conjugates, and by assessing the content of individual conjugated ligands. $M_{\mathrm{w}^{\prime}}$ was calculated as $M_{\mathrm{W}}$ of the precursor increased by the sum of apparent molecular weights of all conjugated ligands. $M_{\mathrm{w}^{\prime}}$ was used to calculate concentrations in all the experiments. The content of fluorescent probes in the conjugates was determined spectrophotometrically (ATTO488 $\varepsilon 502 \mathrm{~nm}=90,000 \mathrm{~L} \cdot \mathrm{mol}^{-1} \cdot \mathrm{cm}^{-1}$ in water; Cy7.5 $\varepsilon 788 \mathrm{~nm}=223,000 \mathrm{~L} \cdot \mathrm{mol}^{-1} \cdot \mathrm{cm}^{-1}$ in water), and the content of biotin was assessed colorimetrically. The amount of conjugated tris-NTA was determined by amino acid analysis (Table 1). Yields of the iBodies $1-3$ were $14 \mathrm{mg}, 14 \mathrm{mg}$, and $40 \mathrm{mg}$, respectively.

To enable complexation of polyhistidine sequences, the tris-NTA targeting ligands must be charged with metal cations (Figure 2). To do so, iBodies 1 and 2 were incubated with either $\mathrm{CoCl}_{2}$ or $\mathrm{NiCl}_{2}$ to charge the NTA groups with $\mathrm{Co}^{2+}$ or $\mathrm{Ni}^{2+}$, respectively. The concentrations of the charged anti-polyHis iBodies were experimentally determined with amino acid analysis to ensure precise comparison of binding properties.

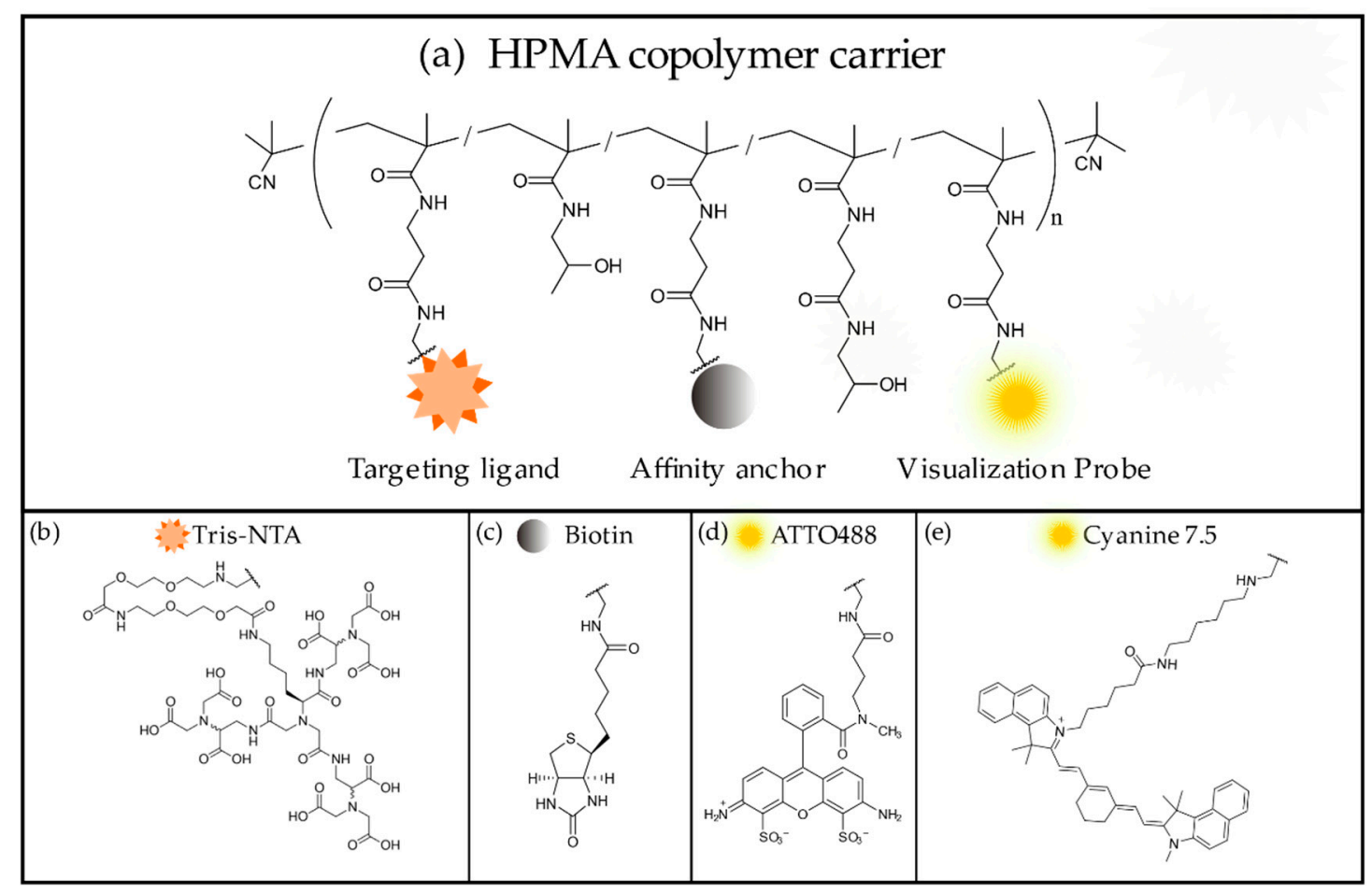

Figure 1. Chemical structure of the $N$-(2-hydroxypropyl) methacrylamide (HPMA) copolymer carrier and its functional ligands. (a) General design of the HPMA copolymer carrier. (b) Tris-nitrilotriacetic acid (tris-NTA) targeting ligand. (c) Biotin affinity anchor. (d) ATTO488 fluorophore ligand (iBody 2). (e) Cyanine 7.5 fluorophore ligand (iBody 1). 


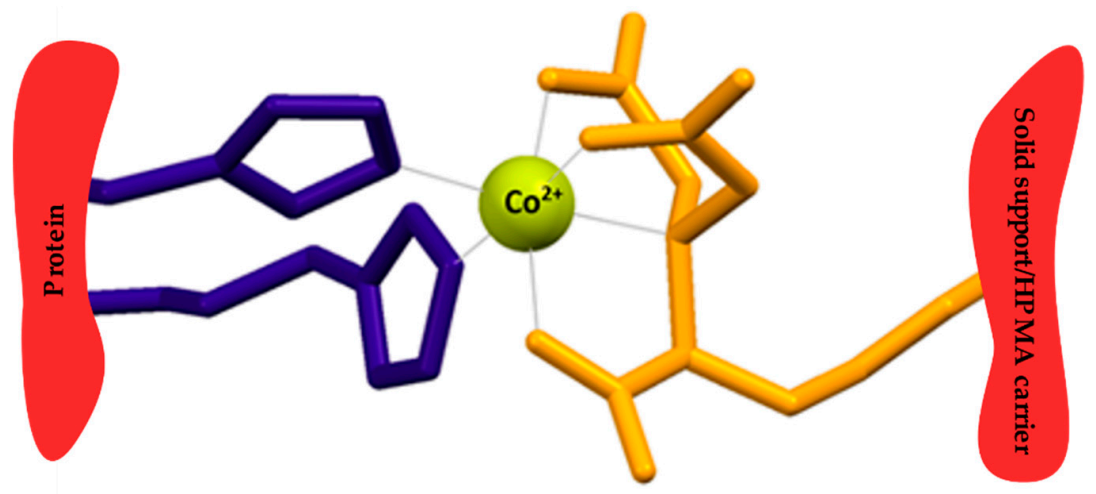

Figure 2. Illustration of $\mathrm{Co}^{2+}$-mediated interaction between histidines and a nitrilotriacetic acid (NTA) moiety. The tris-NTA functional group, which shows higher affinity to His-tag due to the multivalency of NTA [11], is used as a targeting ligand in iBodies.

Table 1. Composition of the anti-polyHis iBodies.

\begin{tabular}{|c|c|c|c|c|c|c|c|c|c|}
\hline Polymer Conjugate & \multicolumn{3}{|c|}{ iBody 1} & \multicolumn{3}{|c|}{ iBody 2} & \multicolumn{3}{|c|}{ iBody 3} \\
\hline Target & \multicolumn{3}{|c|}{ Polyhistidine Sequence } & \multicolumn{3}{|c|}{ Polyhistidine Sequence } & \multicolumn{3}{|c|}{$\begin{array}{c}\text { No Target } \\
\text { (Negative Control) }\end{array}$} \\
\hline$M_{\mathrm{w}}[\mathrm{kDa}]^{*}$ & \multicolumn{3}{|c|}{150} & \multicolumn{3}{|c|}{209} & \multicolumn{3}{|c|}{131} \\
\hline$M_{\mathrm{n}}[\mathrm{kDa}]^{*}$ & \multicolumn{3}{|c|}{112} & \multicolumn{3}{|c|}{150.4} & \multicolumn{3}{|c|}{80.9} \\
\hline$\Xi^{*}$ & \multicolumn{3}{|c|}{1.34} & \multicolumn{3}{|c|}{1.39} & \multicolumn{3}{|c|}{1.62} \\
\hline$M_{\mathbf{w}^{\prime}}[\mathrm{kDa}]^{*}$ & \multicolumn{3}{|c|}{89.4} & \multicolumn{3}{|c|}{98.3} & \multicolumn{3}{|c|}{78.0} \\
\hline & Moiety & $\begin{array}{l}\text { No. Per } \\
\text { Polymer }\end{array}$ & $\mathbf{w t} \%$ & Moiety & $\begin{array}{l}\text { No. Per } \\
\text { Polymer }\end{array}$ & $\mathbf{w t} \%$ & Moiety & $\begin{array}{l}\text { No. Per } \\
\text { Polymer }\end{array}$ & wt $\%$ \\
\hline Targeting Ligand & Tris-NTA & 10.6 & 13.7 & Tris-NTA & 13.7 & 16.2 & - & - & - \\
\hline Fluorophore & Cyanine 7.5 & 2.5 & 2.1 & ATTO488 & 10.2 & 6.6 & ATTO488 & 5.1 & 4.1 \\
\hline Affinity Anchor & Biotin & 10.6 & 3.4 & Biotin & 13.0 & 3.8 & Biotin & 8.2 & 3 \\
\hline
\end{tabular}

${ }^{*} M_{\mathrm{w}}$, weight-average molecular weights; $M_{n}$, number-average molecular weights; $\oslash$, dispersity; $M_{\mathrm{w}^{\prime},} M_{\mathrm{w}}$ of precursor polymer, increased by the sum of apparent molecular weights of all conjugated ligands; No. per polymer, an average number of conjugated ligands per molecule of HPMA polymer carrier.

\subsection{Choice and Establishment of SUMO1 Proteins as a Model System}

To obtain a reliable system of target proteins for use in evaluating the anti-polyHis iBodies, we prepared N-terminally hexa- and deca-His-tagged SUMO1 ubiquitin-like proteins ( $\mathrm{Mw}=12 \mathrm{kDa})$. We chose this protein primarily because of its high stability, high production yields, and the simplicity of in vitro experiments.

The recombinant SUMO1 variants were heterologously expressed in Escherichia coli and purified on nickel-NTA resin (see Supplementary Materials, Table S1 and Figure S1). The proteins showed a high level of purity on SDS-PAGE. Concentrations of both proteins were determined by amino acid analysis. Total yields of $9.1 \mathrm{mg} 6 \times$ His SUMO1 and $13.8 \mathrm{mg} 10 \times$ His SUMO1 per $0.5 \mathrm{~L}$ of culture media were obtained.

\subsection{Determination of Binding Constants of iBodies}

Simple sandwich ELISA was performed to measure the binding constants of iBodies to His-tag and evaluate the differences in strength of cobalt- or nickel-mediated interactions with $6 \times \mathrm{His}$ and $10 \times$ His fusion tags. iBody 1 and iBody 2 charged with either $\mathrm{Co}^{2+}$ or $^{2+}$ cations were bound to immobilized $6 \times$ His- and $10 \times$ His-tagged SUMO1 proteins. Neutravidin-horseradish peroxidase (Neu-HRP) was bound via the biotin anchor of the iBodies and used for luminescent detection. The binding constants are summarized in Table 2; for additional details, see Table S2 and Figure S2 in the Supplementary Materials. 
Table 2. Dissociation constants of anti-polyHis iBodies *.

\begin{tabular}{|c|c|c|c|c|}
\hline Polymer Conjugate & \multicolumn{2}{|c|}{ iBody 1 } & \multicolumn{2}{c|}{ iBody 2 } \\
\hline Mediator Cation & Cobalt & Nickel & Cobalt & Nickel \\
\hline K$_{\mathbf{D}}[\mathbf{n M}] \mathbf{6 \times H i s - t a g}$ & $33 \pm 2$ & $111 \pm 16$ & $10 \pm 1$ & $55 \pm 7$ \\
\hline K$_{\mathbf{D}}[\mathbf{n M}] \mathbf{1 0 \times H i s - t a g}$ & $41 \pm 3$ & $190 \pm 17$ & $25 \pm 9$ & $78 \pm 6$ \\
\hline
\end{tabular}

* Dissociation constants $\left(\mathrm{K}_{\mathrm{D}}\right)$ for binding of the iBodies to $6 \times$ His and 10×His-tagged SUMO1 were determined with ELISA.

All measured $K_{D}$ values were in the nanomolar range. We observed a clear difference in the binding of differently prepared conjugates, sharing the same trends. As expected, based on the literature [3], the interactions of cobalt-charged iBodies with polyhistidine sequences show higher affinities than those mediated by nickel. Furthermore, anti-polyHis iBodies exhibited higher affinity for hexa-His-tagged proteins than for deca-His-tagged proteins. We hypothesize that this might be due to the steric hindrance of the longer tag with the protein. On the other hand, we also used the anti-polyHis iBodies for immobilization of various proteins of much larger sizes than $12 \mathrm{kDa}$ (e.g., immunoreceptors, insulin receptors) and have not observed any differences in binding due to larger sizes of the protein (data not shown).

The $K_{D}$ values for iBody 2 suggest higher affinities in comparison to iBody 1 . This is likely caused by the higher number of conjugated tris-NTA ligands (see Table 1), a general avidity trend that we have observed with iBodies. Higher nonspecific binding, most likely due to the nature of the Cyanine 7.5 fluorophore, was observed for iBody 1 (not shown here, see Figure S2 in the Supplementary Materials).

\subsection{Stability Testing}

To test the stability of the anti-polyHis iBodies, $\mathrm{Co}^{2+}$-charged iBody 1 was incubated at $37^{\circ} \mathrm{C}$ in the dark for 24 months. The solution evaporated during this period, and thus the iBodies had to be redissolved in $\mathrm{H}_{2} \mathrm{O}$. Remarkably, the performance of the anti-polyHis iBodies, which were stored at unfavorable conditions, is comparable to the regularly stored iBodies $\left(-20^{\circ} \mathrm{C}\right)$ as evidenced by results from Western blotting and immunoprecipitation assays.

\subsection{Immobilization of Proteins and Isolation from Cell Lysate}

iBody 1 and iBody 2 charged with cobalt or nickel cations, along with iBody 3 (negative control), were immobilized on prewashed streptavidin agarose beads via their biotin ligands. Additionally, iBody $1 / \mathrm{Co}^{2+}$ used for stability testing at $37^{\circ} \mathrm{C}$ (see above) was included in the experiment. Commercially available anti-His $\mathrm{mAb}$ was immobilized on protein-G sepharose beads via the Fc domain and tested alongside for comparison. To assess the ability of the iBodies to isolate His-tagged proteins from a complex mixture, a eukaryotic cell lysate containing $6 \times$ His- or 10×His-SUMO1 was used for pull-down experiments. The immunoprecipitated proteins were eluted from the beads by incubation in sodium dodecyl sulfate (SDS) buffer at high temperature. The elution fractions were then resolved on SDS-PAGE and stained using the classical silver staining procedure.

All anti-polyHis iBodies tested were able to isolate His-tagged proteins from the cell lysate (Figure 3). Corresponding to the binding constants measured by ELISA, iBodies exhibited stronger affinity to $6 \times$ His-tagged proteins than $10 \times$ His-tagged ones. However, unlike the $\mathrm{mAb}$, the iBodies were able to pull down 10×His-tagged SUMO1, although in low amounts. Interestingly, the different $K_{D}$ values of the same iBody charged with different metal cations did not seem to have an effect on the binding of $6 \times$ His SUMO1. For $10 \times$ His SUMO1, the observed binding with respect to amounts of isolated protein exhibited the opposite trend as observed in other experiments $\left(\mathrm{Ni}^{2+}\right.$-charged iBodies seem to be more effective). We propose that the presence of free metals and reduction agents (such as glutathione) in the cell lysate may influence the formation and stability of the tris-NTA/metal/His-tag complex. 
(a)

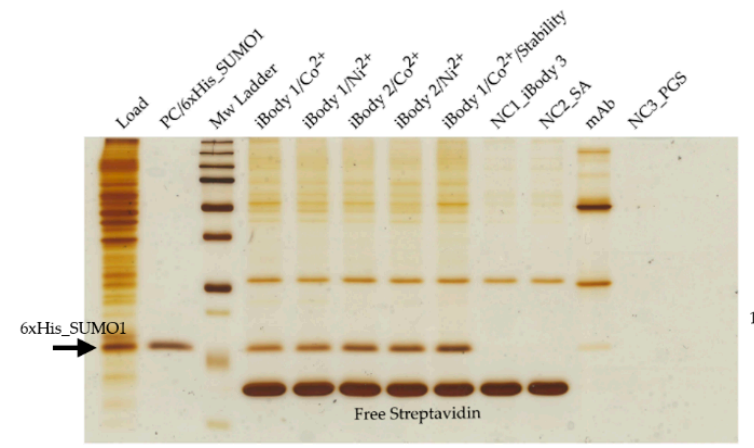

(b)

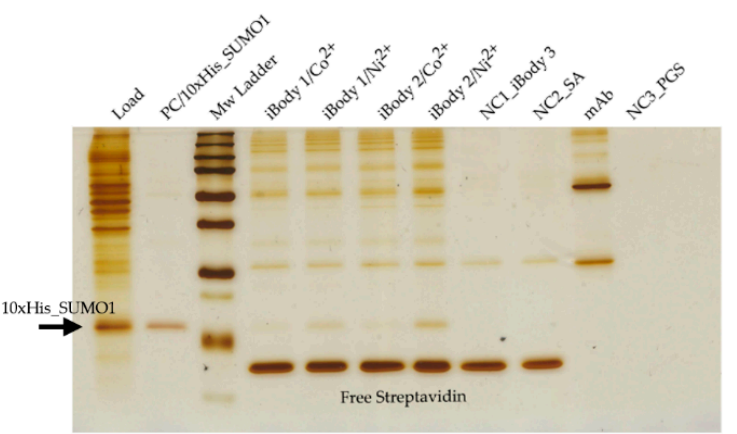

Figure 3. Isolation of $6 x$ or 10xHis-tagged SUMO1 proteins from spiked eukaryotic cell lysate using immobilized anti-polyHis iBodies and anti His-tag antibody. 6x and 10×His-tagged SUMO1 proteins were used as positive controls (PC). iBody 3, blank streptavidin agarose beads (SA) and blank Protein Sepharose beads (PGS) were used as negative controls (NC1, NC2, NC3, respectively). (a) Isolation of $6 \times$ His-tagged SUMO1 protein. (b) Isolation of 10×His-tagged SUMO1 protein.

In comparison to the $\mathrm{mAb}$, the polymer conjugates exhibited a higher background, which is partially caused by the use of streptavidin agarose beads. However, iBodies appear to be superior to the used commercial mAbs with respect to the amounts of the immunoprecipitated protein. Results of the repeated isolation of His-tagged SUMO1 show the reproducibility of the results (Supplementary Figure S3).

\subsection{Visualization of His-Tagged Proteins on Western Blot}

Detection of recombinant proteins on Western blot (WB) is an indispensable part of the protein purification process. To assess the ability of anti-polyHis iBodies to detect His-tagged proteins on WB, two different amounts of $6 \times$ His SUMO1 and 10×His SUMO1 were resolved by SDS-PAGE, transferred to nitrocellulose membranes, and incubated with the corresponding $4 \mathrm{nM}$ iBody solutions. All four iBodies (iBody 1 and 2 charged with cobalt or nickel cations), along with iBody $1 / \mathrm{Co}^{2+} / 37^{\circ} \mathrm{C}$ from stability testing, were evaluated. mAb-CF770, a conjugate of anti-His-tag antibody and a near-IR fluorophore, was used for comparison. As shown in the Figure 4, all iBodies detected the His-tagged SUMO1 proteins. Detection with iBody 1 was in general more effective than with iBody 2 . The signal intensities achieved with commercially available mAb-fluorophore conjugate are superior but still comparable to the detection with the most favorable iBodies. Remarkably, iBody $1 / \mathrm{Co}^{2+}$ was able to detect the proteins even after 24 months of storage at $37^{\circ} \mathrm{C}$. For the quantification of relative Western blot signals, see Figure S4 and Table S3 in the Supplementary Materials. In contrast to the routinely used WB procedure, which commonly employs primary and secondary antibodies, iBodies can detect proteins on WB in a one-step procedure with significantly shorter incubation times of roughly $20 \mathrm{~min}$.

iBody $1 / \mathrm{Co}^{2+}$

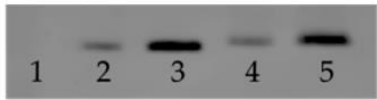

iBody $1 / \mathrm{Ni}^{2+}$

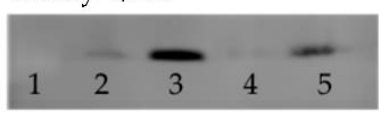

iBody $2 / \mathrm{Co}^{2+}$

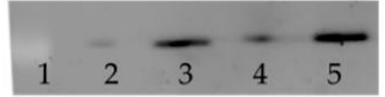

iBody $2 / \mathrm{Ni}^{2+}$

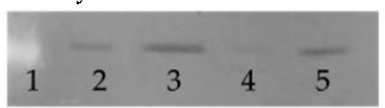

iBody $1 / \mathrm{Co}^{2+} /$ Stability at $37^{\circ}$

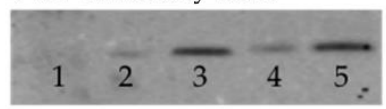

anti-His $\mathrm{mAb} / \mathrm{CF} 770$

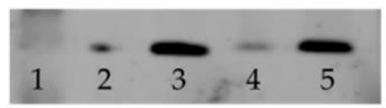

Figure 4. Detection of $6 \times$ His- and 10×His-SUMO1 on Western blot using iBodies and anti-His mAb-CF770 conjugate. Lanes 1-5 in all blots correspond to: molecular weight ladder, $50 \mathrm{ng} 6 \times \mathrm{His}$ SUMO1, 100 ng 6×His SUMO1, 50 ng 10×His SUMO1, and 100 ng 10×His SUMO1. 


\section{Materials and Methods}

\subsection{Preparation of iBody Conjugates}

\subsubsection{General Procedures}

Detailed experimental procedures for the synthesis of the HPMA copolymer precursor and the tris-NTA ligands, as well as the process of ligand conjugation, have been described previously [1]. Generally, the anti-polyHis polymer conjugates iBody 1 and 2 were prepared by reaction of the copolymer precursor poly(HPMA-co-Ma-B-Ala-TT) containing thiazolidine-2-thione reactive groups (TT) along the polymer chain with a mixture of fluorophore molecules (fluorophore- $\mathrm{NH}_{2}$ ), affinity anchors (biotin- $\left.\mathrm{NH}_{2}\right)$, and targeting ligands $\left(\mathrm{NH}_{2}\right.$-tris-NTA) in a desired ratio.

\subsubsection{Synthesis of iBody 1}

Copolymer precursor poly(HPMA-co-Ma-ß-Ala-TT) $\left(15 \mathrm{mg}, 9.96 \times 10^{-6} \mathrm{~mol}\right.$ TT groups; $\left.M_{\mathrm{w}}=64,400 \mathrm{~g} / \mathrm{mol}, M_{\mathrm{n}}=58,500 \mathrm{~g} / \mathrm{mol}, \nexists=1.10\right), \mathrm{NH}_{2}$-tris-NTA $\left(3.0 \mathrm{mg}, 2.36 \times 10^{-6} \mathrm{~mol}\right)$, Cy7.5-amine $\left(1.0 \mathrm{mg}, 1.22 \times 10^{-6} \mathrm{~mol}\right)$ and $\mathrm{N}$-(2-aminoethyl) biotinamide hydrobromide (biotin- $\left.\mathrm{NH}_{2}\right)(1.5 \mathrm{mg}$, $4.08 \times 10^{-6} \mathrm{~mol}$ ) were dissolved in $0.3 \mathrm{~mL}$ of $\mathrm{N}, \mathrm{N}$-dimethylacetamide (DMA). $\mathrm{N}, \mathrm{N}$-diisopropylethylamine (DIPEA) $\left(3.5 \mu \mathrm{L}, 1.53 \times 10^{-5} \mathrm{~mol}\right)$ was added, and the reaction was carried out at room temperature (RT) for $4 \mathrm{~h}$. Then, $2 \mu \mathrm{L}$ of 1-aminopropan-2-ol were pipetted into the mixture to remove residual TT reactive groups, and the reaction was stirred for $10 \mathrm{~min}$. Copolymer conjugate poly(HPMA-co-Ma- $B-$ Ala-TrisNTA-co-Ma-ß-Ala-Cy7.5-co-Ma-ß-Ala-biotin) (iBody 1) was isolated by precipitation into a mixture of acetone:diethyl ether (3:1), filtered off, washed with acetone and diethyl ether and dried in vacuo. Polymer conjugate was purified on a Sephadex LH-20 chromatography column in methanol. Finally, methanol was evaporated, and the conjugate was dissolved in water, purified on a PD-10 column and lyophilized.

\subsubsection{Synthesis of iBody 2}

Copolymer precursor poly(HPMA-co-Ma-ß-Ala-TT) $\left(15.1 \mathrm{mg} ; 1.00 \times 10^{-5} \mathrm{~mol}\right.$ TT groups $\left.M_{\mathrm{W}}=64,400 \mathrm{~g} / \mathrm{mol}, M_{\mathrm{n}}=58,500 \mathrm{~g} / \mathrm{mol}, \oslash=1.10\right)$, tris-NTA $\left(3.5 \mathrm{mg}, 2.75 \times 10^{-6} \mathrm{~mol}\right)$, ATTO $488-\mathrm{NH}_{2}$ $\left(1.69 \mathrm{mg}, 1.97 \times 10^{-6} \mathrm{~mol}\right)$ and $\mathrm{N}$-(2-aminoethyl)biotinamide hydrobromide (biotin- $\left.\mathrm{NH}_{2}\right)(1.63 \mathrm{mg}$, $4.44 \times 10^{-6} \mathrm{~mol}$ ) were dissolved in $0.3 \mathrm{~mL}$ of dimethyl sulfoxide (DMSO). $\mathrm{N}, \mathrm{N}$-diisopropylethylamine (DIPEA) $\left(8.0 \mu \mathrm{L}, 4.58 \times 10^{-5} \mathrm{~mol}\right)$ was then added, and the reaction was carried out for $4 \mathrm{~h}$ at room temperature. Next, $2 \mu \mathrm{L}$ of 1-aminopropan-2-ol was added to remove the residual TT reactive groups, and the reaction was stirred for $10 \mathrm{~min}$. Copolymer conjugate poly(HPMA-co-Ma-ß-Ala-TrisNTAco-Ma-ß-Ala-ATTO488-co-Ma-ß-Ala-biotin) (iBody 2) was isolated by precipitation into a mixture of acetone:diethyl ether (3:1), filtered off, washed with acetone and diethyl ether and dried in vacuo. The polymer conjugate was purified on a Sephadex LH-20 chromatography column in methanol. Finally, methanol was evaporated, and the conjugate was dissolved in water, purified on a PD-10 column and lyophilized.

\subsubsection{Synthesis of iBody 3}

Copolymer precursor poly(HPMA-co-Ma-ß-Ala-TT) $\left(50 \mathrm{mg} ; 3.76 .00 \times 10^{-5} \mathrm{~mol}\right.$ TT groups $\left.M_{\mathrm{w}}=73,000 \mathrm{~g} / \mathrm{mol}, M_{\mathrm{n}}=60,000 \mathrm{~g} / \mathrm{mol}, \oplus=1.22\right)$, ATTO $488-\mathrm{NH}_{2}\left(2.5 \mathrm{mg}, 2.91 \times 10^{-6} \mathrm{~mol}\right)$ and $\mathrm{N}$-(2-aminoethyl)biotinamide hydrobromide (biotin- $\left.\mathrm{NH}_{2}\right)\left(6.0 \mathrm{mg}, 1.63 \times 10^{-5} \mathrm{~mol}\right)$ were dissolved in $0.5 \mathrm{~mL}$ of dimethyl sulfoxide (DMSO). $N, N$-diisopropylethylamine (DIPEA) $\left(6.8 \mu \mathrm{L}, 3.9 \times 10^{-5}\right.$ mol was then added, and the reaction was carried out for $4 \mathrm{~h}$ at room temperature. Next, $3 \mu \mathrm{L}$ of 1-aminopropan-2-ol were added to remove the residual TT reactive groups, and the reaction was stirred for 10 min. Copolymer conjugate poly(HPMA-co-Ma-ß-Ala-ATTO488-co-Ma-ß-Ala-biotin) (iBody 3) was isolated by precipitation into a mixture of acetone:diethyl ether (3:1), filtered off, washed with acetone and diethyl ether and dried in vacuo. The polymer conjugate was purified on a Sephadex 
LH-20 chromatography column in methanol. Finally, methanol was evaporated, and the conjugate was dissolved in water, purified on a PD-10 column and lyophilized.

\subsubsection{Charging Tris-NTA with Metal Cations}

Lyophilized anti-polyHis iBody 1 and iBody 2 were weighed and dissolved in $10 \mathrm{mM} \mathrm{CoCl}$ or $\mathrm{NiCl}_{2}$. Samples were incubated on a rotator at room temperature for $1 \mathrm{~h}$. The solutions were then transferred into dialysis chambers ( $10 \mathrm{kDa}$ cutoff) and allowed to equilibrate in $5 \mathrm{~L}$ of water at $4{ }^{\circ} \mathrm{C}$ for $36 \mathrm{~h}$ with multiple (6x) exchanges of water. After dialysis, the concentrations of the charged anti-polyHis iBodies in solution were experimentally determined by amino acid analysis.

\subsubsection{Determination of the Composition and Molecular Weight of iBodies}

The weight-average molecular weights $\left(M_{\mathrm{w}}\right)$, number-average molecular weights $\left(M_{\mathrm{n}}\right)$, and dispersity $(\boxplus)$ of the polymer precursor and conjugates were determined using a HPLC (Shimadzu, Japan) system equipped with a UV-DAD detector, an Optilab ${ }^{\circledR} \mathrm{NEO}$ differential refractometer, Viscostar III and DAWN-Heleos II multi-angle light scattering (all from Wyatt Technology, USA) detector and a size-exclusion chromatography on a Superose 6 column. The $M_{\mathrm{W}}, M_{\mathrm{n}}$ and $Đ$ were calculated using Astra 7 software. The refractive index increment $\mathrm{dn} / \mathrm{dc}=0.167 \mathrm{~mL} / \mathrm{g}$ was used for calculation. These experiments were conducted with $0.3 \mathrm{M}$ sodium acetate, $\mathrm{pH} 6.5$, buffer at a flow rate of $0.5 \mathrm{~mL} / \mathrm{min}$.

The content of TT reactive groups in the polymer precursors and fluorescent dyes in the conjugates were determined spectrophotometrically (TT $\varepsilon_{305 \mathrm{~nm}}=10,600 \mathrm{~L} \cdot \mathrm{mol}^{-1} \cdot \mathrm{cm}^{-1}$, methanol), (ATTO488 $\varepsilon_{502 \mathrm{~nm}}=90,000 \mathrm{~L} \cdot \mathrm{mol}^{-1} \cdot \mathrm{cm}^{-1}$, water) and $\left(\right.$ Cy7.5 $\varepsilon_{788 \mathrm{~nm}}=223,000 \mathrm{~L} \cdot \mathrm{mol}^{-1} \cdot \mathrm{cm}^{-1}$, water). The biotin content was determined colorimetrically using the HABA/Avidin Reagent Kit (Sigma-Aldrich) the results were corrected for the effect of ATTO488 absorbance at $500 \mathrm{~nm}$.

The content of tris-NTA in iBodies 1 and 2 was determined by amino acid analysis of the hydrolyzed polymer conjugates $\left(6 \mathrm{M} \mathrm{HCl}, 115^{\circ} \mathrm{C}, 18 \mathrm{~h}\right.$ in a sealed vial). A reversed-phase column (Chromolith ${ }^{\circledR}$ HighResolution RP-18e, $100 \times 4.6 \mathrm{~mm}$ ) (Merck, Germany), following pre-column derivatization with 2,3-naphthalenedicarboxaldehyde (NDA)/ $\mathrm{NaCN}$ (source of cyanide ions), and a fluorescence detector (excitation at $229 \mathrm{~nm}$, emission at $490 \mathrm{~nm}$ ) were used for the analysis. Gradient elution with $10 \%-100 \%$ solvent $\mathrm{B}(300 \mathrm{~mL}$ of $0.17 \mathrm{M}$ sodium acetate and $700 \mathrm{~mL}$ of methanol) was performed for $35 \mathrm{~min}$ at a flow rate of $1.0 \mathrm{~mL} / \mathrm{min}$. Solvent A was $0.05 \mathrm{M}$ sodium acetate buffer, $\mathrm{pH} 6.5$.

\subsection{Expression and Purification of the His-Tagged SUMO1 Proteins}

\subsubsection{Plasmid Preparation and Cloning}

We selected the $p E T-15 b$ expression plasmid, which encodes a $6 \times$ His-tag, for production of recombinant SUMO1 proteins with $6 \times$ His and $10 \times$ His fusion tags. We used as a template the pHisTEV30a-SUMO1 plasmid encoding the protein of interest (along with a Tobacco Etch Viru (TEV) protease cleavage site and $10 \times$ His cleavage) prepared in-house.

First, the protein coding sequence was amplified from $p H i s T E V 30 a-S U M O 1$ using Taq polymerase and primers $5^{\prime}$-aaacatatgtctgaccaggag- $3^{\prime}\left(\mathrm{Fwd}, \mathrm{T}_{\mathrm{m}}=57^{\circ} \mathrm{C}\right.$ ) and $5^{\prime}$-aaactcgagctaaccccc- $3^{\prime}$ (Rev, $\mathrm{T}_{\mathrm{m}}=59^{\circ} \mathrm{C}$ ) including $\mathrm{NdeI}$ and XhoI restriction sites. To obtain the $p E T-15 b$ vector coding for $6 \times$ His-tagged SUMO1 protein, the amplified PCR product was cleaved with XhoI and NdeI and ligated into $p E T-15 b$ via corresponding restriction sites. Next, the newly cloned $p E T-15 b-6 \times H i s S U M O 1$ plasmid served as a template for PCR amplification of the $10 \times$ His-tagged SUMO1 protein coding sequence the using primers $5^{\prime}$-taccatgggcagcagccatcatcatcatcatcatcatcatcatcacagca- $3^{\prime}\left(\mathrm{Fwd}, \mathrm{T}_{\mathrm{m}}=89.8^{\circ} \mathrm{C}\right)$ and $5^{\prime}$-atctcgagctaacccccegtttgttcctgataaacttcaatcacatcttc- $3^{\prime}\left(\operatorname{Rev}, \mathrm{T}_{\mathrm{m}}=83.0^{\circ} \mathrm{C}\right)$ including NcoI and XhoI restriction sites. The obtained PCR product was cleaved and ligated into $p E T-15 b$ via NcoI and XhoI restriction sites, yielding $p E T-15 b-10 \times$ HisSUMO1 vector. To verify the sequences of the obtained plasmids, control digestion reactions and Sanger sequencing were performed. 


\subsubsection{Expression of Recombinant SUMO Proteins}

Recombinant SUMO1 proteins with N-terminally fused polyhistidine affinity tags of different lengths were expressed in E. coli. BL21-DE3-RIL competent cells were first transformed with the two prepared plasmids, $p E T-15 b-6 \times$ HisSUMO1 and $p E T-15 b-10 \times$ HisSUMO1, then plated on LB agar containing ampicillin $(100 \mu \mathrm{g} / \mu \mathrm{L})$ and allowed to grow at RT for $48 \mathrm{~h}$. A single colony was picked from each plate and separately transferred into liquid media containing ampicillin $(8 \mathrm{~mL} \mathrm{LB}, 100 \mu \mathrm{g} / \mathrm{mL}$ ampicillin). After allowing the transformed bacterial cells to grow at $37^{\circ} \mathrm{C}$ on a shaker for $12 \mathrm{~h}$, each of the pre-grown cultures was used to inoculate $500 \mathrm{~mL}$ of LB media. The cultures were allowed to grow at $16^{\circ} \mathrm{C}$ on a shaker. After cultures reached an $\mathrm{OD}_{600}$ of 0.6 , protein expression was induced by addition of $0.4 \mathrm{mM}$ Isopropyl $\mathrm{B}$-D-1-thiogalactopyranoside (IPTG)and allowed to progress overnight. Afterwards, both samples were centrifuged, and the pellets were resuspended in lysis buffer $(50 \mathrm{mM}$

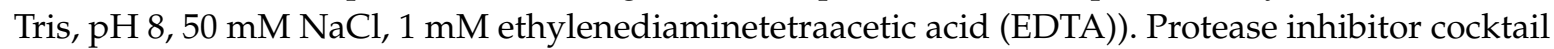
(Sigma Aldrich, Czech Republic) was added, and the samples were homogenized with an Avestin EMULSIFLEX C3 instrument (ATA Scientific, Australia).

\subsubsection{Purification of Recombinant His-SUMO Proteins}

Cell lysates from expression cultures were collected and used for purification on nickel-NTA resin. Washed resin $(0.5 \mathrm{~mL})$ was added to each lysate, and the samples were allowed to incubate for $2 \mathrm{~h}$ at RT. The resin with bound proteins was washed three times $(50 \mathrm{mM}$ Tris, $\mathrm{pH} 8.0,300 \mathrm{mM}$ $\mathrm{NaCl}, 20 \mathrm{mM}$ imidazole), and the proteins were eluted using buffer with a high concentration of imidazole (50 mM Tris, $\mathrm{pH}$ 8.0, $300 \mathrm{mM} \mathrm{NaCl}, 250 \mathrm{mM}$ imidazole). Elution fractions containing the desired products were transferred into Tris-buffered saline (TBS; $50 \mathrm{mM}$ Tris, $\mathrm{pH} 7.6,137 \mathrm{mM} \mathrm{NaCl}$ ) by dialysis. All fractions (flow-throughs and elutions) were collected during the procedure and used for analysis with silver-stained SDS-PAGE and Western blotting (Supplementary Materials, Figure S1, Table S1). Amino acid analysis was performed to determine the concentrations of both proteins with high precision.

\subsection{Testing the Anti-polyHis iBodies}

\subsubsection{Determining the Binding Constants of iBodies with ELISA}

To measure the binding constants of iBodies to His-tag and evaluate the differences in strength of cobalt- or nickel-mediated interactions with $6 \times$ His and $10 \times$ His fusion tags, simple sandwich ELISA was performed in a 96-well, flat-bottom, MaxiSorp, nontransparent plate (Thermo Fisher Scientific, Czech Republic). SUMO1 protein (20 ng per well) was immobilized directly onto the plate $(1 \mathrm{~h}, \mathrm{RT})$ and blocked with $1 \% \mathrm{BSA}$ in TBS $\left(4^{\circ} \mathrm{C}, \mathrm{O} / \mathrm{N}\right)$. iBody 1 or iBody 2 were then bound to the protein in a concentration series (60 min, RT) (see details in Table S2). In the last layer of the sandwich, neutravidin-HRP was bound via biotin and used for detection. Between binding steps of each new species, wash steps were performed, i.e., $4 \times$ TBS with $0.1 \%$ Tween 20 (TBST). Immediately after addition of the HRP substrate, luminescence at $428 \mathrm{~nm}$ was measured on a TECAN Infinite M1000 instrument (TECAN, Germany).

\subsubsection{Immobilization on iBodies and Isolation of His-SUMO1 Proteins from Cell Lysate}

iBody $1\left(\mathrm{Ni}^{2+}\right.$ or $\mathrm{Co}^{2+}$ charged), iBody 1 (stability testing, $\mathrm{Co}^{2+}$ charged), iBody $2\left(\mathrm{Ni}^{2+}\right.$ or $\mathrm{Co}^{2+}$ charged), iBody 3 (negative control), and anti-His-tag mAb (Sigma Aldrich, Czech Republic) were immobilized on specialized beads and used for affinity pull-down of SUMO1 proteins. The iBodies were immobilized on streptavidin agarose Ultra Performance beads (Solulink, USA) via their biotin ligands. The $\mathrm{mAb}$ was immobilized on protein-G sepharose beads 4 fast flow (GE Healthcare, USA) via the Fc domain. Aliquots of $300 \mu \mathrm{L}$ of each $150 \mathrm{nM}$ species to be bound were incubated with $12 \mu \mathrm{L}$ prewashed beads (TBS, $4{ }^{\circ} \mathrm{C}, \mathrm{O} / \mathrm{N}$ ) for $90 \mathrm{~min}$ at $\mathrm{RT}$. 
Eukaryotic cell lysate (total protein concentration $\sim 7 \mathrm{mg} / \mathrm{mL}$ ) was $4 \mathrm{x}$ diluted in TBST and spiked with either $6 \times$ His SUMO1 or $10 \times$ His SUMO1 (final SUMO1 concentration of $20 \mathrm{ng} / \mu \mathrm{L}$ ). Each immobilized species was then mixed with the spiked cell lysate (each sample containing $4 \mu \mathrm{g}$ of SUMO1) and allowed to incubate $(1.5 \mathrm{~h}, \mathrm{RT})$. Immunoprecipitated proteins were eluted from resins by incubation in $30 \mu \mathrm{L}$ SDS buffer $\left(10 \mathrm{~min}, 98^{\circ} \mathrm{C}\right)$. Each of the samples from pull-down $(12 \mu \mathrm{L})$ along with negative controls (iBody 3 , blank streptavidin agarose beads and blank protein-G sepharose beads) and positive controls (spiked cell lysate and purified SUMO1) were separated on a $16 \%$ polyacrylamide gel (SDS-PAGE, $200 \mathrm{~V}, 70 \mathrm{~min}, 4^{\circ} \mathrm{C}$ ) and visualized by silver staining.

\subsubsection{Visualization of His-Tagged Proteins on Western Blot}

First, $5 \mu \mathrm{L}$ and $10 \mu \mathrm{L}$ (corresponding to $50 \mathrm{ng}$ and $100 \mathrm{ng}$ ) of $6 \times$ His- and 10×His-SUMO1 were loaded onto $16 \%$ polyacrylamide gels and resolved by SDS-PAGE ( $200 \mathrm{~V}, 70 \mathrm{~min}, 4{ }^{\circ} \mathrm{C}$ ). Proteins were then transferred onto a nitrocellulose membrane (BioRad, Czech Republic) using routine WB procedure (100 V, 60 min). After blotting, membranes were blocked with a TBS/0.5\% Casein Blocker (CB) solution (60 $\mathrm{min}, \mathrm{RT})$. To detect the proteins, membranes were incubated in iBody incubation buffer (TBS, $0.05 \% \mathrm{CB}, 1 \mathrm{mM}$ imidazole, $0.05 \%$ SDS, $0.3 \%$ Tween) containing $4 \mathrm{nM}$ iBody 1 or 2 . For detection with mAb-CF770 (Sigma-Aldrich, Czech Republic), $4 \mathrm{nM}$ antibody in TBST solution was used. After 20 min incubation at RT, blots were washed four times with TBST and allowed to dry. A LI-COR Odyssey CLx instrument (LI-COR Biotechnology, USA) was used for visualization of NIR fluorophore conjugates (iBody 1 and mAb-CF770). A Typhoon FLA 9500 instrument (GE Healthcare, USA) was used to visualize the proteins detected with ATTO488 carrying conjugates (iBody 2).

\section{Conclusions}

Our biomimetic polymeric conjugates offer a new approach to protein immobilization and detection. Here, we demonstrate that iBodies can be used as antibody mimetics to detect and bind His-tagged proteins in common biochemical applications. Furthermore, we demonstrate that iBodies are extremely stable and at least as effective as commonly used anti-His-tag antibodies. In contrast to antibodies, iBodies are effective even after two years of storage at high temperature. By replacing antibodies with polymer conjugates, routine procedures can be simplified and significantly shortened. Additionally, we are exploring further uses of anti-polyHis iBodies, including immobilization of whole proteins via their His-tag and applications for such conjugates.

Most importantly, the iBody platform is extremely flexible. The properties of the conjugates are highly dependent on the nature and number of functional ligands and thus can be adjusted depending on the intended use of iBodies. This work is focused on applications with His-tagged proteins, but, in theory, iBodies can be designed for visualization and immobilization of any protein with known ligands.

\section{Patents}

iBody technology is protected by patents US10114014 (B2), US10302632 (B2), EP3245513(B1), EP3245514(B1), AU2016207125(B2), AU2016207126(B2), CA2970913(C) and CA2970845(C).

Supplementary Materials: The following are available online at http://www.mdpi.com/2073-4344/9/12/1011/s1, Table S1: Overview of samples collected during purification of the $6 \times$ His- and $10 \times \mathrm{His}-\mathrm{SUMO} 1$ proteins and amounts loaded onto $16 \%$ polyacrylamide gels used for subsequent silver staining and WB analyses, Figure S1: (a) Analysis of His-SUMO1 protein purification process: results of silver staining, (b) Analysis of His-SUMO1 protein purification process: results of Western blotting, Table S2: Concentration series of iBodies used for KD determination with ELISA, Figure S2: (a) Graphical representation of binding of iBody $1 / \mathrm{Co}^{2+}$ to His-tagged SUMO1 proteins measured with ELISA, (b) Graphical representation of binding of iBody $1 / \mathrm{Ni}^{2+}$ to His-tagged SUMO1 proteins measured with ELISA, (c) Graphical representation of binding of iBody $2 / \mathrm{Co}^{2+}$ to His-tagged SUMO1 proteins measured with ELISA, (d) Graphical representation of binding of iBody $2 / \mathrm{Ni}^{2+}$ to His-tagged SUMO1 proteins measured with ELISA, Figure S3: (a) Repetition of the immunoprecipitation experiments/pull-down of $6 \times$ His-tagged SUMO1, (b) Repetition of the immunoprecipitation experiments/pull-down of $10 \times$ His-tagged 
SUMO1, Figure S4: Relative quantification of the Western blotting results, Table S3: Summary of the relative signals on Western blots.

Author Contributions: Methodology, iBodies testing, data validation, writing—original draft preparation, J.B.; synthesis and analysis of tris-NTA, A.Š.; synthesis and analysis of polymer precursors and iBodies, V.Š. and L.K.; writing—review and editing, J.K., P.Š. and T.E.; supervision and funding acquisition, J.K., P.Š., T.K., T.E.

Funding: This research was funded by the European Regional Development Fund; OP RDE (No.CZ.02.1.01/0.0/0.0/16_019/0000729), by the Ministry of Industry and Trade (Czech Republic), projects FV10490 and FV10370, by the Czech Science Foundation (No. 19-10280S), by the Ministry of Education, Youth and Sports of CR within the National Sustainability Program II, Project BIOCEV-FAR LQ1604, by the project "BIOCEV-Biotechnology and Biomedicine Centre of the Academy of Sciences and Charles University" (CZ.1.05/1.1.00/02.0109) and by the by Charles University Grant Agency (No. 1670119).

Acknowledgments: We would like to thank to K. Sramkova and J. Starkova for technical support and R. Soucek for performing amino acid analysis.

Conflicts of Interest: The authors declare no conflict of interest.

\section{References}

1. Gaberc-Porekar, V.; Menart, V. Perspectives of immobilized-metal affinity chromatography. J. Biochem. Biophys. Methods 2001, 49, 335-360. [CrossRef]

2. Chaga, G.S. Twenty-five years of immobilized metal ion affinity chromatography: Past, present and future. J. Biochem. Biophys. Methods 2001, 49, 313-334. [CrossRef]

3. Wegner, S.V.; Spatz, J.P. Cobalt(III) as a stable and inert mediator ion between NTA and His6-tagged proteins. Angew. Chem. 2013, 52, 7593-7596. [CrossRef] [PubMed]

4. Ueda, E.K.; Gout, P.W.; Morganti, L. Current and prospective applications of metal ion-protein binding. J. Chromatogr. A 2003, 988, 1-23. [CrossRef]

5. Goding, J.W. Monoclonal Antibodies: Principles and Practice, 3rd ed.; Academic Press Limited: London, UK, 1996.

6. Marx, V. Finding the right antibody for the job. Nat. Methods 2013, 10, 703-707. [CrossRef] [PubMed]

7. Debeljak, N.; Feldman, L.; Davis, K.L.; Komel, R.; Sytkowski, A.J. Variability in the immunodetection of His-tagged recombinant proteins. Anal. Biochem. 2006, 359, 216-223. [CrossRef] [PubMed]

8. Sacha, P.; Knedlík, T.; Schimer, J.; Tykvart, J.; Parolek, J.; Navrátil, V.; Dvořáková, P.; Sedlák, F.; Ulbrich, K.; Strohalm, J.; et al. iBodies: Modular Synthetic Antibody Mimetics Based on Hydrophilic Polymers Decorated with Functional Moieties. Angew. Chem. 2016, 55, 2356-2360. [CrossRef] [PubMed]

9. Dvorakova, P.; Busek, P.; Knedlik, T.; Schimer, J.; Etrych, T.; Kostka, L.; Stollinová Šromová, L.; Subr, V.; Sacha, P.; Sedo, A.; et al. Inhibitor-Decorated Polymer Conjugates Targeting Fibroblast Activation Protein. J. Med. Chem. 2017, 60, 8385-8393. [CrossRef] [PubMed]

10. Simon, P.; Knedlik, T.; Blažková, K.; Dvořáková, P.; Brezinova, A.; Kostka, L.; Subr, V.; Konvalinka, J.; Sacha, P. Identification of Protein Targets of Bioactive Small Molecules Using Randomly Photomodified Probes. ACS Chem. Biol. 2018, 13, 3333-3342. [CrossRef] [PubMed]

11. Huang, Z.; Hwang, P.; Watson, D.S.; Cao, L.; Szoka, F.C., Jr. Tris-nitrilotriacetic acids of subnanomolar affinity toward hexahistidine tagged molecules. Bioconjug. Chem. 2009, 20, 1667-1672. [CrossRef] [PubMed]

(C) 2019 by the authors. Licensee MDPI, Basel, Switzerland. This article is an open access article distributed under the terms and conditions of the Creative Commons Attribution (CC BY) license (http://creativecommons.org/licenses/by/4.0/). 